\title{
Electromagnetic Reduction of Plasma Density During Atmospheric Reentry and Hypersonic Flights
}

\author{
Michael Keidar* \\ The George Washington University, Washington, D.C. 20052 \\ and \\ Minkwan Kim $\ddagger$ and Iain D. Boyd $\$$ \\ University of Michigan, Ann Arbor, Michigan 48109 \\ DOI: $\underline{10.2514 / 1.32147}$
}

\begin{abstract}
As a vehicle reenters or travels through the atmosphere at hypersonic velocities, the shock-heated air surrounding the vehicle becomes weakly ionized. This plasma layer causes an important systems operation problem known as communications blackout or radio blackout. At sufficiently high plasma density, the plasma layer either reflects or attenuates radiowave communications to and from the vehicle. In this paper, we study the application of electric and magnetic fields to reduce the plasma density. Specifically, an $E \times B$ crossed-field configuration is proposed. Both analytical and numerical results suggest that significant reduction of the plasma density is possible at large altitudes. For instance, plasma density reduction by a factor of 10 is predicted in the case of $81 \mathrm{~km}$ and a magnetic field of about 0.1 T. Theoretical results suggest that significant reduction of the plasma density is possible, enabling radio communication across the plasma layer. The benefit of the reduced plasma density in terms of electromagnetic wave absorption across the plasma layer is estimated.
\end{abstract}

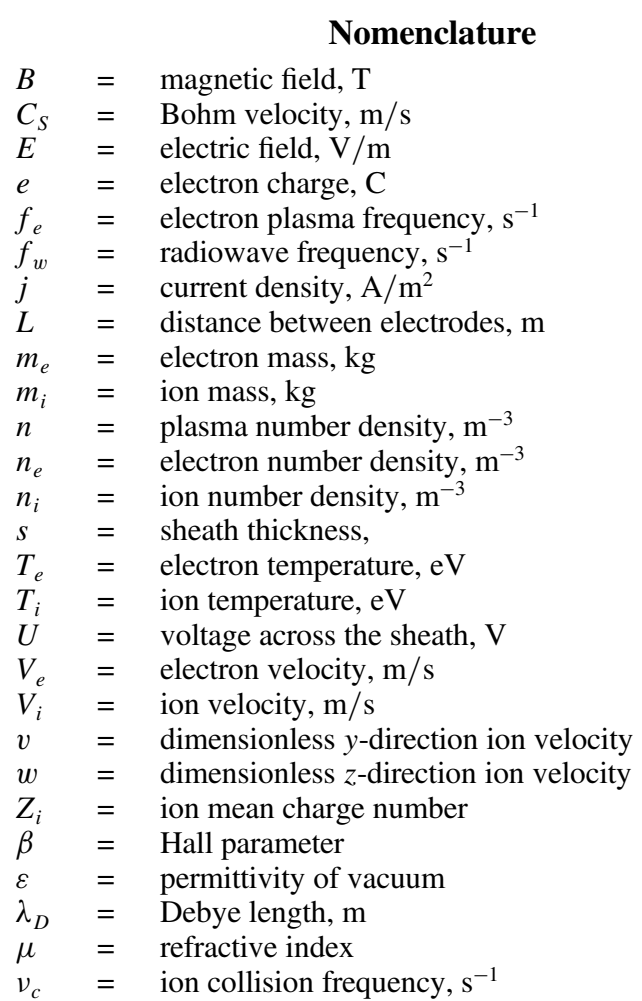

Received 14 May 2007; revision received 6 September 2007; accepted for publication 26 September 2007. Copyright $@ 2007$ by the American Institute of Aeronautics and Astronautics, Inc. All rights reserved. Copies of this paper may be made for personal or internal use, on condition that the copier pay the $\$ 10.00$ per-copy fee to the Copyright Clearance Center, Inc., 222 Rosewood Drive, Danvers, MA 01923; include the code 0022-4650/08 $\$ 10.00$ in correspondence with the CCC.

*Assistant Professor, Department of Mechanical \& Aerospace Engineering; keidar@gwu.edu. Senior Member AIAA.

Graduate Student, Department of Aerospace Engineering. Student Member AIAA.

†Professor, Department of Aerospace Engineering. Associate Fellow AIAA.

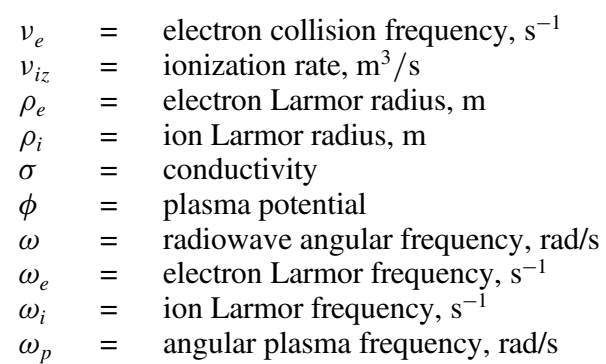

Subscripts

$\begin{array}{lll}e & =\text { electron } \\ i & =\text { ion } \\ \text { th } & =\text { thermal } \\ 0 & =\text { initial value }\end{array}$

I. Introduction

W HEN a spacecraft enters the Earth's atmosphere or a vehicle travels through the atmosphere at hypersonic velocities, a shock wave is formed in front of the vehicle, causing the air to be compressed and heated. The shock wave converts much of the vehicle's kinetic energy into heat and therefore increases the air temperature. As a result, the air molecules become dissociated and ionized. Thus, in the reentry of spacecraft into the Earth's atmosphere, a plasma layer is created due to the ionization of neutral particles by this shock wave heating. Similarly, conditions for plasma formation are created during hypersonic flight. The plasma layer that is formed around the leading edge of the spacecraft is sometimes referred to as the plasma sheath. This plasma layer has an important impact on vehicle operation because it prevents normal telemetry transmission. The same difficulties apply to information transmitted from space probes and other scientific data instruments. The density of the plasma layer is about $10^{15}$ to $10^{19} \mathrm{~m}^{-3}$, and this density is high enough to cause so-called radio blackout [1-5]. For instance, during the blackout, radiowaves emitted from the reentry vehicle cannot reach a ground station because they are reflected against the plasma layer. Therefore, there is a need to develop strategies for propagating telemetry through a plasma layer. Consequently, it is extremely important to have a clear 
understanding of this plasma-layer phenomenon to select optimum system requirements.

In general, two methods of plasma control are applicable for addressing the radio blackout problem: passive control and active control. Passive techniques include change of the leading-edge geometries to decrease the plasma density and allow data to be transmitted through the plasma. Sharply pointed reentry vehicles are surrounded by a much thinner plasma sheath than that surrounding blunted reentry vehicles. However, a sharply pointed reentry vehicle has a reduced payload capability and increased aerodynamic heating problems compared with those of a blunted reentry vehicle [6]. Active technologies usually involve tailoring of the plasma flow by creating virtual geometries. In addition, injection of electrophilic liquids or gases into the shock layer [7] or application of remote antenna assemblies [8] were considered. Alternative solutions were also proposed in the past, such as communication through the plasma via Raman interactions that are based on the nonlinear properties of the plasma [9]. However, the last method may have several severe limitations, such as collisional damping, sensitivity to plasma parameter nonuniformities, and very short range of resonance for three-wave interactions.

On the other hand, if properly produced near the antenna, magnetic fields can create a "window" in the reentry plasma through which electromagnetic waves can be propagated. The magnetic field lines must be oriented such that the electrons are tightly bound to them through gyration and do not respond to the electric field component of the electromagnetic wave [10]. Recent numerical simulations showed that magnetic fields modify wave dispersion under reentry plasma conditions and that the radiowaves can penetrate the reentry plasma mainly by the whistler mode [2]. Among the mechanisms of active plasma control that have been studied, the use of a magnetic field seems to be the most promising for its possibility to tailor the plasma layer during the communication. Similar magnetohydrodynamic (MHD) effects were used to control supersonic flows and scramjet inlets [11]. Calculations demonstrated that an MHD system could bring the oblique shock back to the cowl lip when flying at Mach numbers higher than those for which the scramjet inlet was optimized. However, the effectiveness of a magnetic field for reducing the density in the reentry vehicle plasma layer has not yet been studied in detail. In particular, there has been no investigation into the effects of different magnetic field configurations. In addition, an application of crossed electric and magnetic field $E \times B$ leads to some interesting phenomena, such as electron drift, and the possibility of maintaining a strong electric field in a quasi-neutral plasma [12]. Ion acceleration in this electric field may lead to significant reduction of plasma density.

Active experiments in space are very expensive and therefore few have been performed to study details of plasma-sheath evolution during reentry, with the notable exception of the RAM-C flights conducted in the late 1960s [13-15]. These three flight experiments used Langmuir probes and microwave instruments to determine the plasma density around a conical reentry vehicle. Plasma densities in the range of $10^{15}$ to $10^{19} \mathrm{~m}^{-3}$ were measured over an altitude range from 90 to $25 \mathrm{~km}$. These experiments yielded data that may be useful in helping to identify strategies for solving the blackout problem. Before resorting to new flight experiments on this problem, laboratory experiments should be conducted to test new concepts. However, because the idea to use magnetic control of the plasma density in the sheath of a reentering vehicle is a completely untested concept, numerical simulations of the plasma-sheath creation and development under the influence of applied magnetic fields represents the most appropriate initial approach to this problem. To determine the best configuration of the magnetic field, one needs to understand the parameters of the reentry plasma, such as the profiles of plasma density and electron temperature near the vehicle surface. For this reason, calculations of the flowfield around a reentry vehicle and plasma generation were performed.

Usually, radio blackout occurs when the plasma frequency of the plasma layer around the reentry vehicle exceeds the frequency of the radiowaves $f_{w}$ emitted from the vehicle: $f_{e}>f_{w}$, where $f_{e}$ is the electron plasma frequency [16]:

$$
f_{e}=\frac{\omega_{e}}{2 \pi} \approx 9 \times 10^{3} n_{e}^{\frac{1}{2}} \mathrm{~Hz}
$$

where $n_{e}$ is the plasma density in $\mathrm{cm}^{-3}$. Therefore, detailed knowledge of the plasma density profile is necessary to establish the range of communication signal frequency that is affected.

When $E \times B$ fields are applied, it is expected that the plasma density distribution will be affected, depending on the magnitudes and profiles of the magnetic and electric fields. Numerical simulations can help to estimate this effect and determine the optimal magnetic field configuration for creating the lowest plasma density near the radio antenna. The alleviation of the communication blackout problem requires a thorough and accurate knowledge of the reentry plasma properties to apply any technique in the optimal way. For this reason, physically accurate numerical simulations of the flowfield are required.

This paper is organized as follows. In Sec. II, we present results of the flowfield analysis followed by a description of a model of the hypersonic plasma layer in the $E \times B$ fields. Two regimes of plasmaoptic approximation and MHD approach are described in Secs. III and IV, respectively. In Sec. $\underline{\mathrm{V}}$, an electrostatic mitigation technique is described. In Sec. VI, the benefit of the reduced plasma density in terms of electromagnetic wave absorption across the plasma layer is estimated.

\section{Hypersonic Flowfield Analysis}

The direct simulation Monte Carlo (DSMC) code MONACO, developed at the University of Michigan, was applied to compute the hypersonic flow around the full geometry of the RAM-C vehicle $[14,15]$. We used MONACO to compute the flowfield for the highest altitude conditions from RAM-C of $81 \mathrm{~km}$ and the medium altitude of $71 \mathrm{~km}$. A computational fluid dynamics (CFD) code was applied to compute the hypersonic flow around the RAM-C vehicle in the low-altitude cases at $61 \mathrm{~km}$ and below. The CFD code solves the Navier-Stokes equations using a point-implicit method on general unstructured meshes. A five-species $\left(\mathrm{N}_{2}, \mathrm{O}_{2}, \mathrm{NO}, \mathrm{N}, \mathrm{O}\right)$ reacting-air chemistry model is employed. Electron number densities are computed at each point in the flowfield using Saha relations, along with input from the computed neutral species' number densities and temperatures. More details about hypersonic flow simulations can be found in $[17,18]$.

The calculated temperature and density distributions for species of interest $(\mathrm{O}, \mathrm{N}$, and electrons) are presented in Figs. $\underline{1}$ and $\underline{2}$ at an altitude of $61 \mathrm{~km}$. The predicted electron density is in the range of $10^{17}-10^{19} \mathrm{~m}^{-3}$, depending on the location along the vehicle. The calculated plasma density is compared with the measured data from the RAM-C experiment. Because only maximum plasma densities are available from the experiment, we compare the maximum predicted plasma density with the corresponding experimental data.

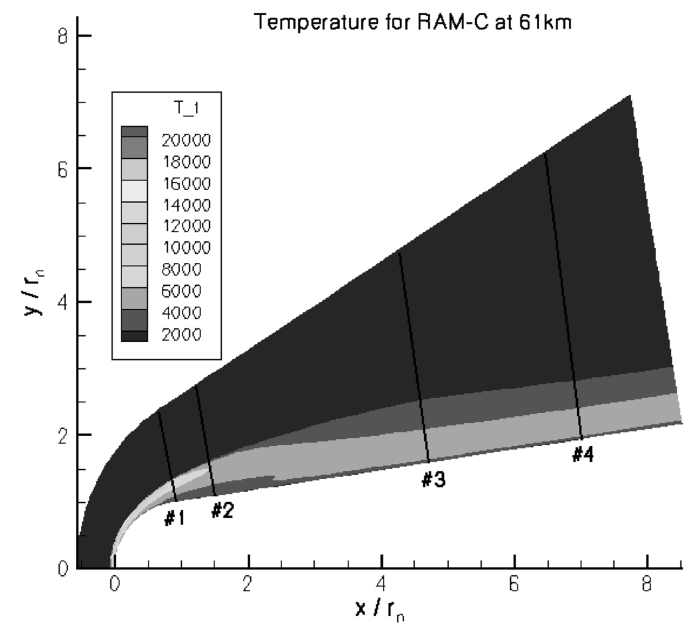

Fig. 1 Contours of translational temperature (K) predicted by the CFD code at $61 \mathrm{~km}$. 


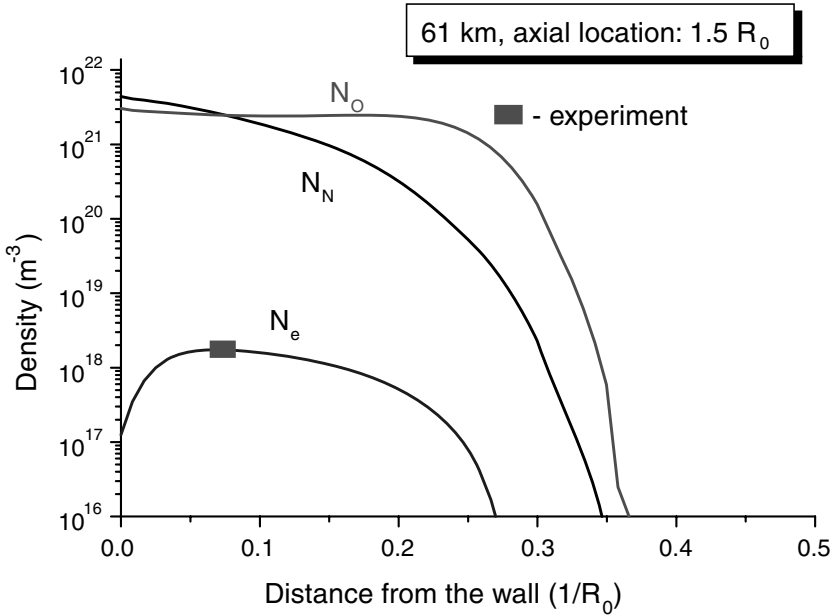

Fig. 2 Density profiles at $61 \mathrm{~km}$ as a function of distance from the wall at axial location $1.5 R_{o}\left(R_{o}\right.$ is the nose radius). Comparison with peak electron density from the experimental data for RAM-C experiment $([14,15])$. Maximum measured plasma density is also indicated.

One can see that generally good agreement is found between the predicted electron density and the measured values. According to our model predictions, the plasma density peak is about $(0.1-0.2) R_{o}\left(R_{o}\right.$ is the nose radius) away from the wall, depending on the position along the vehicle and the altitude. The plasma peak is located closer to the wall $\left(\sim 0.05 R_{o}\right)$ in the case of $61 \mathrm{~km}$, with an axial position of $4.5 R_{o}$.

\section{Plasma-Optic Regime of an $E \times B$ Layer}

Plasma acceleration across the $E \times B$ layer is similar to that in a Hall-effect thruster, in which a large electric field can be maintained in the quasi-neutral plasma across the magnetic field [19]. Schematically the magnetic layer is shown in Fig. $\underline{3}$. The following general assumptions are made:

1) The $E \times B$ layer is quasi neutral.

2) There is no ionization in the $E \times B$ layer.

3) $T_{e}$ is constant.

4) The magnetic field only has an $X$ component: $B=B_{x}$.

Our study suggested that two limiting cases are possible: the plasma-optic regime and the MHD regime. As a first approach, we concentrated on the plasma-optic regime [i.e., a partially magnetized plasma (ions are unmagnetized)]. In this case, the main issue is the maintenance of a strong electric field across a magnetic field. Ions are accelerated in the electric field and the plasma density decreases. In this regime, the ion-neutral collisions are very important. In the highdensity case (below 61-km altitude), we can still expect ion acceleration, but a larger magnetic field is required. In this case, ion-

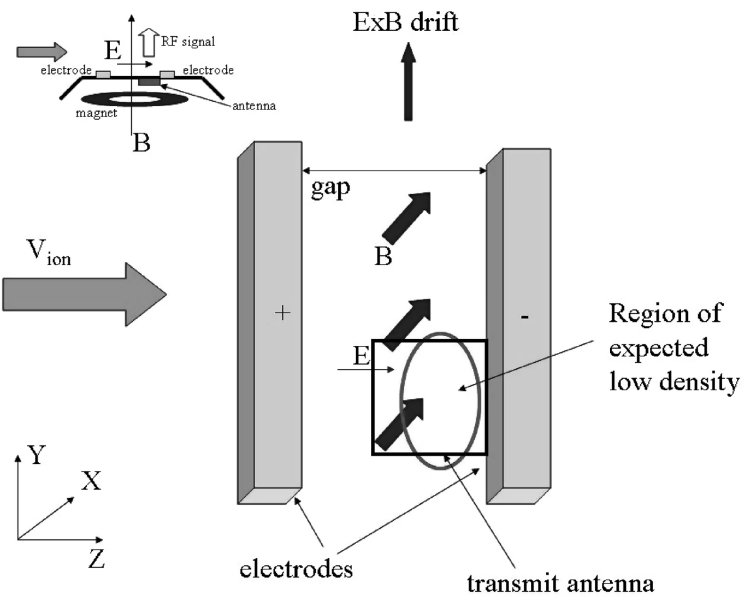

Fig. 3 Schematics of the $E \times B$ layer. neutral coupling becomes important and ions should be considered magnetized. This is the so-called MHD regime, which will be considered at low altitude.

Let us consider conditions of a partially magnetized plasma in which electrons are magnetized and ions are unmagnetized (i.e., $\left.\rho_{e} \ll L \ll \rho_{i}\right)$, where $\rho_{e}$ and $\rho_{i}$ are the Larmor radii for the electrons and ions, respectively, and $L$ is the distance between the electrodes. Thus, a simplified one-dimensional model of the magnetized layer can be developed based on a hydrodynamic model for a Hall thruster [19-21]. Under the considered conditions, the $E \times B$ layer configuration leads to an electron drift in the $E \times B$ direction and provides plasma quasi neutrality across the layer. As a result, a significant electric field can be maintained across the magnetic field. This causes ion acceleration, leading to plasma density decrease.

The following system of equations can be used to describe the effect of the $E \times B$ field in this case:

$$
\begin{gathered}
n m_{i}\left(\boldsymbol{V}_{i} \cdot \nabla \boldsymbol{V}_{i}\right)=e n \boldsymbol{E}-n m_{i} v_{c} \boldsymbol{V}_{i} \\
0=-e n\left(\boldsymbol{E}+\boldsymbol{V}_{e} \times B\right)-k T_{e} \nabla n-n m_{e} v_{e}\left(\boldsymbol{V}_{e}-\boldsymbol{V}_{i}\right) \\
\nabla \cdot(n u)=v_{i z} n_{e}
\end{gathered}
$$

It is assumed that neutrals are at rest, so that the effect of the ionneutral drag is maximized. We start by obtaining the analytical solution of the system $(\underline{1}-\underline{3})$.

\section{A. Analytical Solution}

In component form, Eqs. (1- $\underline{-})$ read

$$
\begin{array}{r}
V_{i z} \frac{\mathrm{d} V_{i y}}{\mathrm{~d} z}=-v_{c} V_{i y} \\
V_{i z} \frac{\mathrm{d} V_{i z}}{\mathrm{~d} z}=\frac{e}{m_{i}}\left\{-\frac{k T_{e}}{e} \frac{\mathrm{d} \log n}{\mathrm{~d} z}+B V_{i y}\right. \\
\left.-\beta_{e} B V_{i z}-\left(\frac{\beta_{e} B}{e n}+\frac{1}{\sigma}\right) j_{z}\right\}-v_{c} V_{i z}
\end{array}
$$

We use the following dimensionless variables:

$$
w=\frac{V_{i z}}{C_{S}}, \quad v=\frac{V_{i y}}{C_{S}}, \quad \zeta=\frac{z}{C_{S} / \omega_{i}}, \quad \delta=\frac{j_{z} / e n}{C_{s}}
$$

After normalization, we arrive at the following system of equations:

$$
w v^{\prime}=-\beta_{i} v
$$

$$
w w^{\prime}=-\delta\left(\beta_{e}+\frac{1}{\beta_{e}}\right)-\beta_{e} w+v-\beta_{i} w-\frac{w^{\prime}}{w}
$$

Typically, the axial velocity $V_{i z}$ is much larger than $V_{i y}$, and so we can ignore the $v$ term on the right-hand side of Eq. (7). Thus, one can arrive at the following equation:

$$
\left(1+\frac{1}{w^{2}}\right) w^{\prime}=-\frac{\delta}{w}\left(\beta_{e}+\frac{1}{\beta_{e}}\right)-\left(\beta_{e}+\beta_{i}\right)
$$

For a high-altitude high-magnetic-field condition, parameter $\delta$ becomes small enough so that usually

$$
\frac{\delta}{w}\left(\beta_{e}+\frac{1}{\beta_{e}}\right) \ll\left(\beta_{e}+\beta_{i}\right)
$$

Integrating the preceding equation yields the following: 


$$
w+\frac{1}{w}-\left(w_{o}+\frac{1}{w_{0}}\right)=-\left(\beta_{e}+\beta_{i}\right) \zeta
$$

Solving for normalized velocity $w$ yields

$w=\frac{1}{2}\left[w_{0}+\frac{1}{w_{0}}-\left(\beta_{e}+\beta_{i}\right) \zeta\right] \pm \sqrt{\frac{\left[w_{0}+\frac{1}{w_{0}}-\left(\beta_{e}+\beta_{i}\right) \zeta\right]^{2}}{4}-1}$

The positive sign in Eq. (10) corresponds to ion deceleration and the negative sign corresponds to ion acceleration. In our case, we are interested in the situation with ion acceleration, so that only one solution has physical meaning:

$$
\begin{aligned}
w & =\frac{1}{2}\left[w_{0}+\frac{1}{w_{0}}-\left(\beta_{e}+\beta_{i}\right) \zeta\right] \\
& -\sqrt{\frac{\left[w_{0}+\left(1 / w_{0}\right)-\left(\beta_{e}+\beta_{i}\right) \zeta\right]^{2}}{4}-1}
\end{aligned}
$$

Simulations show that the ionization term in Eq. (3) does not affect the results and thus may be omitted in this analysis. Thus, the plasma density distribution can be calculated as follows:

$$
N=\frac{w_{0}}{\frac{1}{2}\left[w_{0}+\frac{1}{w_{0}}-\left(\beta_{e}+\beta_{i}\right) \zeta\right]-\sqrt{\frac{\left[w_{0}+\left(1 / w_{0}\right)-\left(\beta_{e}+\beta_{i}\right) \zeta\right]^{2}}{4}-1}}
$$

\section{B. Simulation Result}

In this section, we present results of the numerical solution of the system in Eqs. (1-3). Plasma density values [22-25] are listed in Table 1 for various altitudes. In addition, we list values of plasma densities employed in our simulation.

The plasma and neutral densities are taken from the MONACO simulation for $81 \mathrm{~km}$ and from the CFD simulation for $61 \mathrm{~km}$, respectively. To illustrate the effect of collisionality, we show plasma density reduction as a function of altitude in Fig. 4 . We define the plasma density reduction as a ratio of the plasma density near the negative electrode to the initial plasma density in the flowfield. One can see that at $61 \mathrm{~km}$, the reduction is relatively small, and at $41 \mathrm{~km}$, the reduction is negligible under the considered conditions. This simulation result says that density reduction is a function of altitude. For the case of $41 \mathrm{~km}$, the density reduction is very low even at the $0.5-\mathrm{T}$ condition. Therefore, we will seek another approach for plasma mitigation at low altitude, as described in the next section.

The reduction in plasma density with current density as a parameter is shown in Fig. 5. The effect of the current density on the plasma density reduction for $81-\mathrm{km}$ conditions is shown in Fig. 6a. One can see that a higher-current density across the $E \times B$ layer leads to stronger plasma density reduction effect. In the case of $61 \mathrm{~km}$, shown in Fig. 5 b, the same density reduction can be achieved by increasing the current density by factor of 2 . For instance, in the case

Table 1 Boundary condition for plasma density dependent on the altitude

\begin{tabular}{ccc}
\hline \hline Altitude, $\mathrm{km}$ & Initial plasma density & Reference \\
\hline 61 & $10^{20} \mathrm{~m}^{-3}$ & {$[\underline{22}]$} \\
71 & $4 \times 10^{19} \mathrm{~m}^{-3}$ & {$[\underline{22}]$} \\
81 & $2 \times 10^{18} \mathrm{~m}^{-3}$ & {$[\overline{22}]$} \\
64.6 & $6 \times 10^{17} \mathrm{~m}^{-3}$ & {$[\underline{23}]$} \\
61 & $2.5 \times 10^{19} \mathrm{~m}^{-3}$ & {$[\overline{23}]$} \\
61 & $7 \times 10^{20} \mathrm{~m}^{-3}$ & {$[\underline{24}]$} \\
41 & $8 \times 10^{19} \mathrm{~m}^{-3}$ & {$[\underline{24}]$} \\
81 & $10^{18} \mathrm{~m}^{-3}$ & {$[\underline{25}]$} \\
71 & $2 \times 10^{19} \mathrm{~m}^{-3}$ & {$[\underline{25}]$} \\
61 & $10^{20} \mathrm{~m}^{-3}$ & {$[\underline{25}]$} \\
81 & $10^{18} \mathrm{~m}^{-3}$ & {$[\underline{25}]$} \\
61 & $10^{19} \mathrm{~m}^{-3}$ & These simulations \\
41 & $10^{21} \mathrm{~m}^{-3}$ & These simulations \\
\hline \hline
\end{tabular}

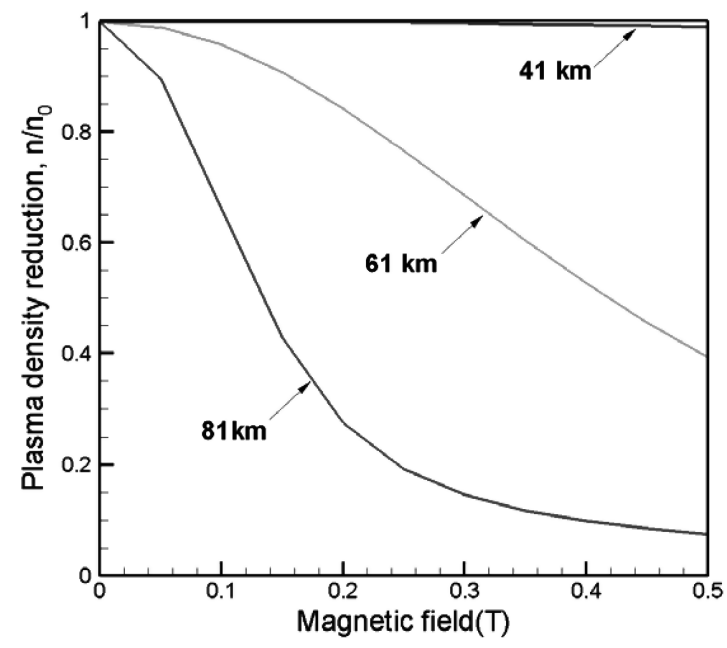

Fig. 4 Plasma density reduction for three different altitude conditions. For $81 \mathrm{~km}$, plasma density is $n_{e}=10^{18} \mathrm{~m}^{-3}$ and neutral density is $n_{a}=10^{21} \mathrm{~m}^{-3}$. For $61 \mathrm{~km}, n_{e}=10^{19} \mathrm{~m}^{-3}$ and $n_{a}=10^{22} \mathrm{~m}^{-3}$. For $41 \mathrm{~km}$, $n_{e}=10^{21} \mathrm{~m}^{-3}$, and $n_{a}=10^{24} \mathrm{~m}^{-3}$. Current density $j=200 \mathrm{~A} / \mathrm{m}^{2}$.

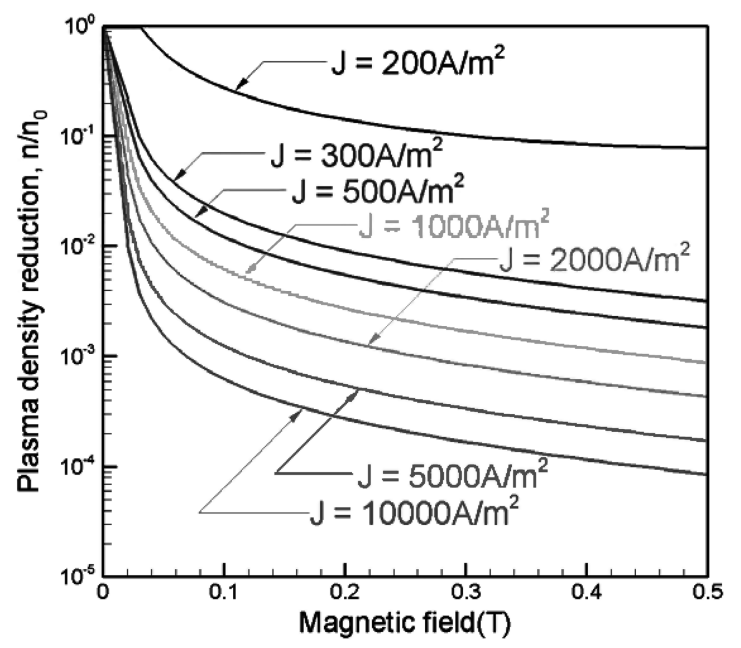

a)

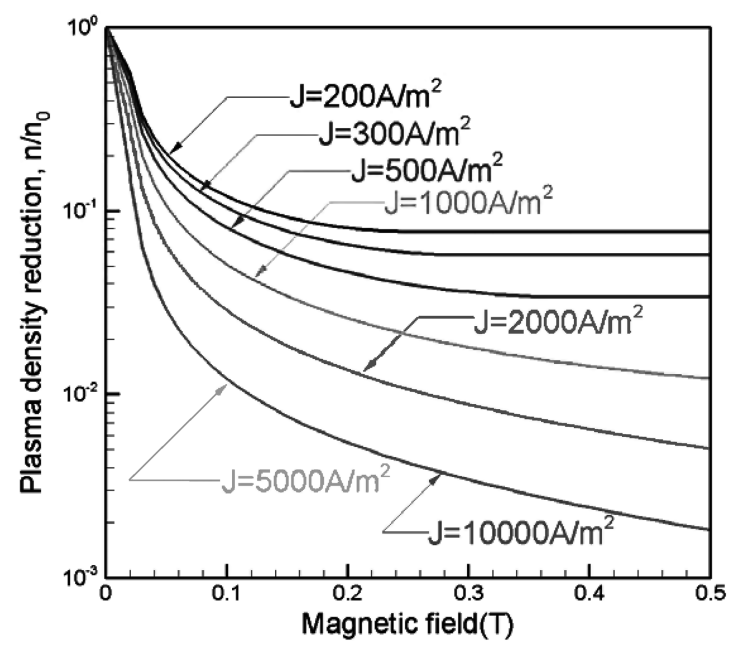

b)

Fig. 5 Plasma density reduction as a function of magnetic field with current density as a parameter; altitude is a) $81 \mathrm{~km}$ with interelectrode distance $L=10 \mathrm{~cm}$ and initial ion velocity $V=1000 \mathrm{~m} / \mathrm{s}$ and b) $61 \mathrm{~km}$ with a 10-cm system length. 


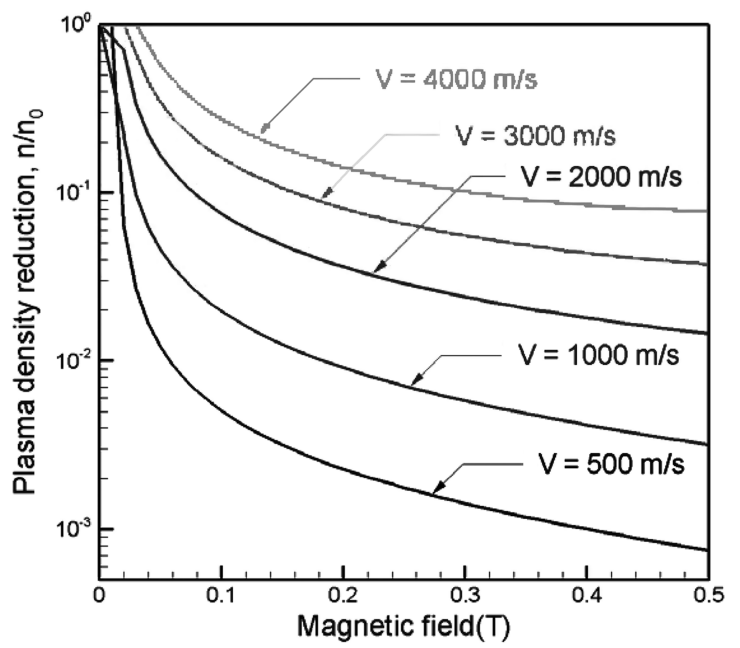

a)

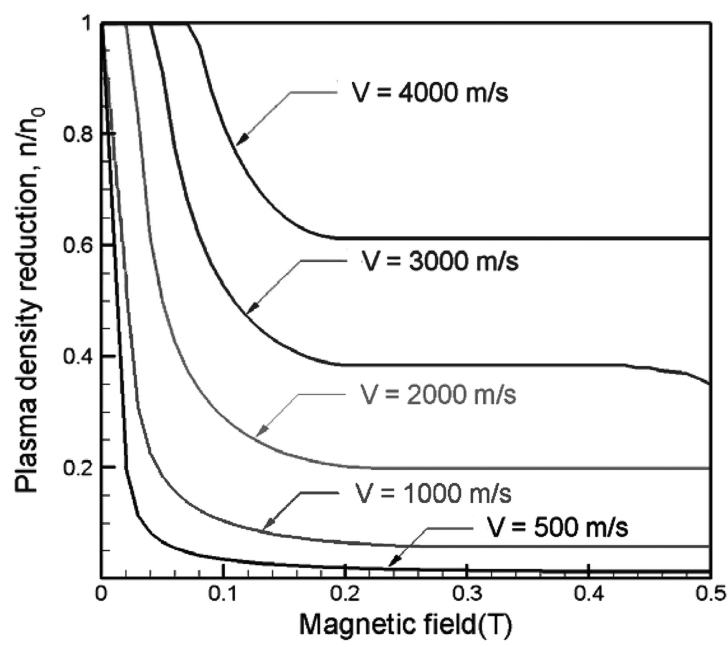

b)

Fig. 6 Plasma density reduction as a function of magnetic field with ion velocity as a parameter; altitude is a) $81 \mathrm{~km}$ with $L=10 \mathrm{~cm}$ and b) $61 \mathrm{~km}$ with $L=10 \mathrm{~cm}$.

of $81 \mathrm{~km}\left(j=10^{3} \mathrm{~A} / \mathrm{m}^{2}\right.$ and $\left.B=0.5 \mathrm{~T}\right)$, the plasma density is reduced by two orders of magnitude, which may be sufficient to allow radio communication.

High-current density leads to a stronger $E \times B$ field, and as a result, plasma density reduction becomes stronger. However, in a real system, the current density cannot be controlled. Instead, the potential drop across the gap can be set. The high-current density means that a large potential drop is required. The potential drop is also affected by the initial velocity $\mathrm{V}$ and system length $L$.

The initial velocity also affects the plasma density reduction, as shown in Fig. 6, but this effect is weaker than that of the current density. It follows from Fig. 6 that a lower initial velocity gives a higher plasma density reduction.

The voltage drop across the $E \times B$ layer is shown in Figs. 7a and $7 \mathrm{~b}$. The voltage across the $E \times B$ layer could be limited by technical, weight, or other considerations. Such limitations would impose limiting conditions on the maximum current density allowed.

By limiting the potential drop at the level of $10 \mathrm{kV}$, one can find a solution map, as shown in Fig. 8 . It can be seen that a higher ion velocity generally requires a higher-current density to obtain a plausible solution.

\section{MHD Regime}

In this section, we describe theoretical analysis of the $E \times B$ layer in the case of strong ion-neutral coupling (i.e., in the MHD regime).

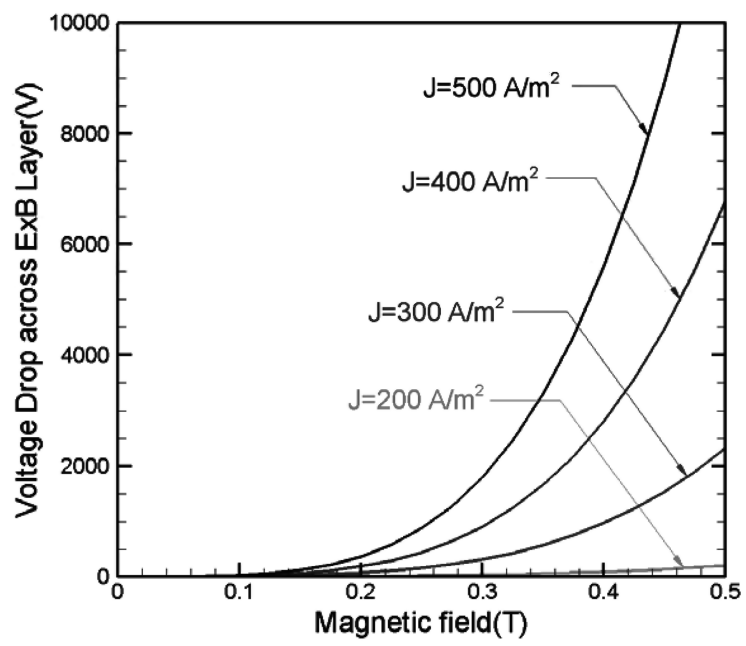

a)

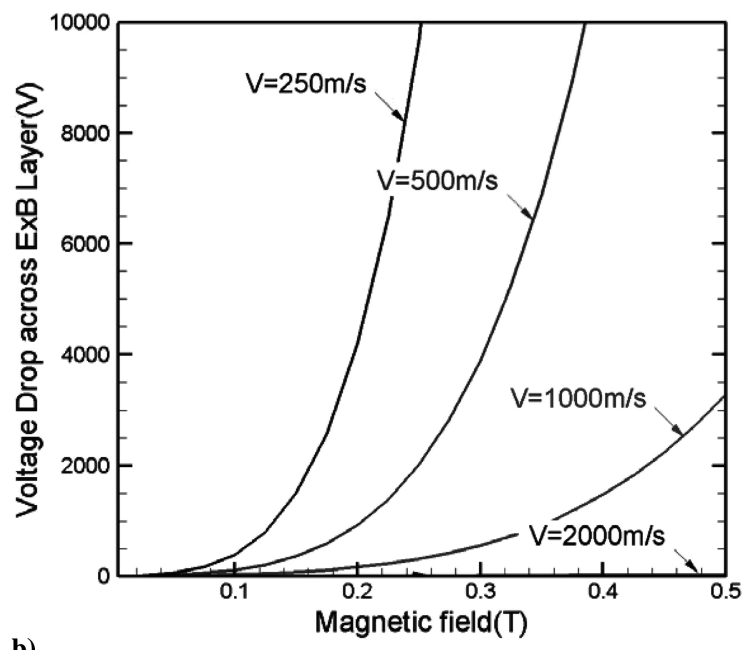

Fig. 7 Voltage drop across the $E \times B$ layer in the case of $81 \mathrm{~km}$ with a) current density as a parameter; $V=1000 \mathrm{~m} / \mathrm{s}$ and b) ion velocity as a parameter.

\section{A. Analytical Solution}

We assume that the ion velocity and electron velocity are the same, because the current density in the $y$ direction is small enough to be neglected in comparison with the current density in the $z$ direction.

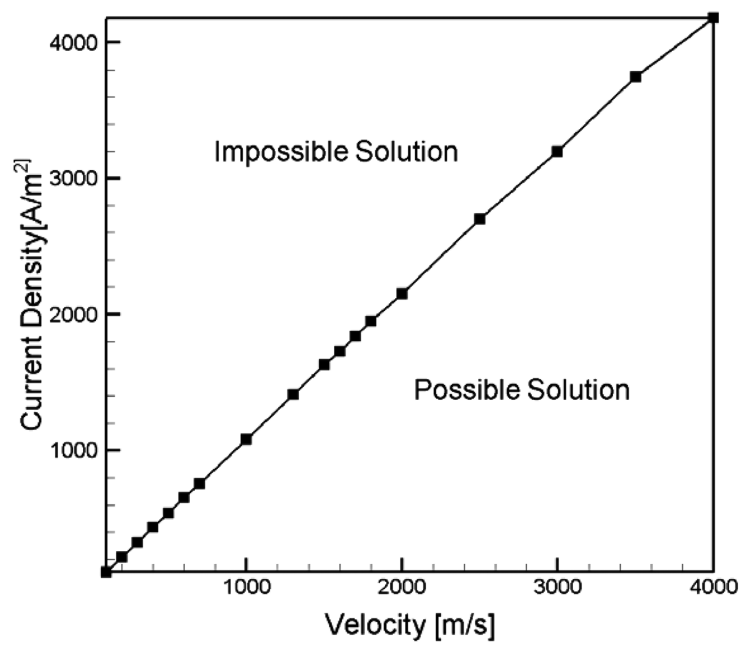

Fig. 8 Possible solution map for $81-\mathrm{km}$ altitude with $L=10 \mathrm{~cm}$ and $1000 \mathrm{~m} / \mathrm{s}$ initial velocity. The condition is for a plasma density reduction $n / n_{o}<0.1$. 
Under these conditions, the collision drag terms are negligible. Because we are looking for density reduction along the $y$ direction, it is assumed that the plasma only has variation along the $y$ direction.

$$
\begin{gathered}
V_{y} \frac{\mathrm{d} V_{y}}{\mathrm{~d} y}=\frac{J_{z} B_{0}}{m_{i} n}-\frac{T_{e}}{m_{i}} \frac{1}{n} \frac{\mathrm{d} n}{\mathrm{~d} y} \\
V_{y} \frac{\mathrm{d} V_{z}}{\mathrm{~d} y}=-\frac{J_{y} B_{0}}{m_{i} n}
\end{gathered}
$$

In addition, we use the continuity equation:

$$
\nabla \cdot(n V)=0
$$

Introduce dimensionless variables as follows:

$$
v=\frac{V_{y}}{V_{y 0}} ; \quad N=\frac{n}{n_{0}}
$$

where $V_{y 0}$ is the initial $y$-direction velocity, and $n_{0}$ is the initial plasma density:

$$
\frac{\mathrm{d}}{\mathrm{d} y}\left(n V_{y}\right)=0
$$

so that the dimensionless equation $v N=1$.

All spatial variables are normalized by $L_{y}$, which is the system length in the $y$ direction $\left(Y=y / L_{y}\right)$. In this case, our MHD system will have the following form:

$$
\begin{gathered}
\frac{V_{y 0}^{2}}{L_{y}} v v^{\prime}=\frac{J_{z} B_{0}}{m_{i} n_{0}} v+\frac{T_{e}}{m_{i} L_{y}} v^{-1} v^{\prime} \\
-\frac{V_{y 0}^{2}}{L_{y}} \frac{1}{N^{3}} N^{\prime}=\frac{J_{z} B_{0}}{m_{i} n_{0}} \frac{1}{N}-\frac{T_{e}}{m_{i} L_{y}} \frac{1}{N} N^{\prime} \\
-N^{\prime}=\frac{J_{z} B_{0} L_{y}}{m_{i} n_{0} V_{y 0}^{2}} N^{2}-\frac{T_{e}}{m_{i} V_{y 0}^{2}} N^{2} N^{\prime}
\end{gathered}
$$

We use additional dimensionless variables:

$$
E_{\mathrm{th}}=\frac{T_{e}}{m_{i} V_{y 0}^{2}}
$$

is the dimensionalized electron thermal energy and

$$
E_{\text {current }}=\frac{J_{z} B_{0} L_{y}}{n_{0}} \frac{1}{m_{i} V_{y 0}^{2}}
$$

is the dimensionalized $J \times B$ energy.

Physically, $E_{\text {th }}$ means the ratio between the thermal energy of electron to the kinetic energy, and $E_{\text {current }}$ means the ratio of the ampere force energy per particle to the kinetic energy. Using these new variables, the system of equations (17-19) reads as

$$
\begin{gathered}
-N^{\prime}=E_{\text {current }} N^{2}-E_{\mathrm{th}} N^{2} N^{\prime} \\
\left(E_{\mathrm{th}} N^{2}-1\right) N^{\prime}=E_{\text {current }} N^{2} \\
E_{\mathrm{th}} N^{\prime}+\left(\frac{1}{N}\right)^{\prime}=E_{\text {current }}
\end{gathered}
$$

Integration of the last equation leads to

$$
E_{\text {th }}(N-1)+\left(\frac{1}{N}-1\right)=E_{\text {current }} Y
$$

After some transformation, this equation reads

$$
E_{\mathrm{th}} N^{2}-\left(E_{\mathrm{th}}+1+E_{\text {current }} Y\right) N+1=0
$$

Solving for plasma density $N$, one can arrive at the following:

$$
N=\frac{\left(E_{\mathrm{th}}+1+E_{\text {current }} Y\right) \pm \sqrt{\left(E_{\mathrm{th}}+1+E_{\text {current }} Y\right)^{2}-4 E_{\mathrm{th}}}}{2 E_{\mathrm{th}}}
$$

The second term of the right-hand side should have a negative sign, because density decreases along the $y$ direction. So our final analytical solution is

$$
N=\frac{\left(E_{\text {th }}+1+E_{\text {current }} Y\right)-\sqrt{\left(E_{\text {th }}+1+E_{\text {current }} Y\right)^{2}-4 E_{\text {th }}}}{2 E_{\text {th }}}
$$

The ratio between $E_{\text {current }}$ and $E_{\text {th }}$ determines the density reduction:

$$
\frac{E_{\mathrm{current}}}{E_{\mathrm{th}}}=\frac{J_{z} B_{0} L_{y}}{n_{0} T_{e}}
$$

This ratio increases linearly with current density $J_{z}$. Analytical predictions for plasma density reduction are shown in Figs. $\underline{9}$ and $\underline{10}$ as a function of the magnetic field.

In the high-altitude condition, the MHD model predicts nearly the same result as the plasma-optic approximation. However, MHD predicts a much stronger plasma density reduction in the low-altitude case. This fact tells us that the collision term becomes important at low altitude. The analytical solution suggests that the plasma density reduction could be significant if the current density is larger than $5000 \mathrm{~A} / \mathrm{m}^{2}$, as shown in Fig. 9. One can see that the plasma density reduction is also affected by the length of the system (Fig. 10). Changing two variables (current density and system length) makes it possible to obtain the desired plasma density reduction. It should be pointed out that in the plasma-optic regime, the plasma density reduction is small, as shown in Sec. III. On the other hand, substantial density reduction is predicted in $\mathrm{MHD}$ regime.

\section{B. Numeral Calculations and Comparison with Analytical Results}

In this section, we present the results of the numerical calculations of Eqs. (13) and (14) and compare these results with the analytical predictions. The system of equations (13), (14), and (16) was solved numerically using the Runge-Kutta method. In these calculations, an initial potential distribution was assumed to be linearly distributed between the electrodes. The iteration procedure is used to calculate the final potential distribution. The plasma density sharply changes near the electrode, and thus we used a fine grid size in this region. A grid sensitivity study was performed to determine the required grid size for accurate simulation.

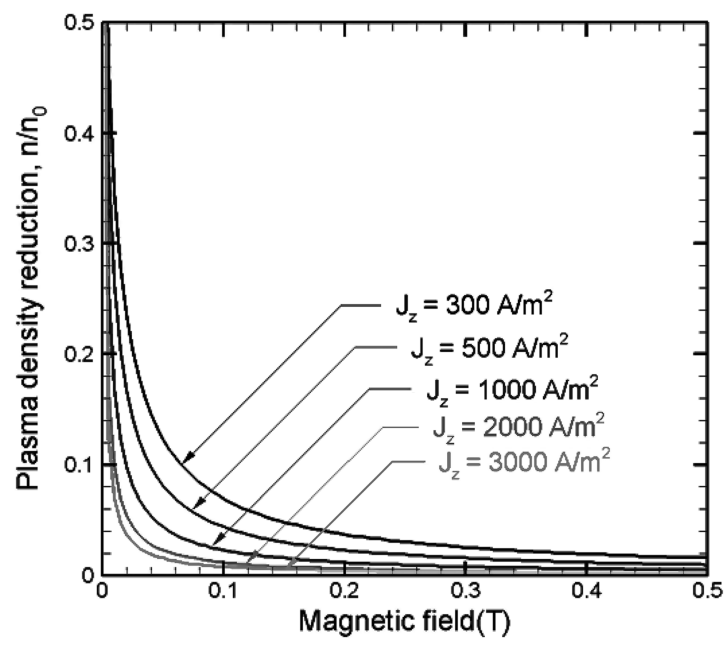

Fig. 9 Analytical solution result for $81 \mathrm{~km}$ with a 10-cm system length. 


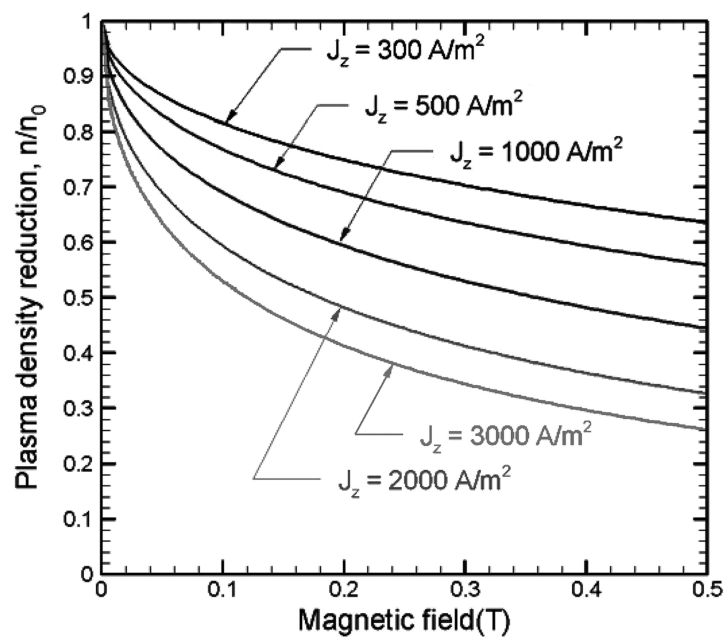

Fig. 10 Analytical solution result for $41 \mathrm{~km}\left(300 \mathrm{~A} / \mathrm{m}^{2}\right)$ with a 10-cm system length.

The comparison is shown in Figs. 11a-11c. One can see that the difference between the analytical and numerical solutions is not very large for the entire span of altitudes between $41-81 \mathrm{~km}$. The deviation of the analytical prediction from numerical simulation becomes larger at low-altitude conditions. Mainly, the difference is due to the fact that the analytical solution does not account for the ion-electron drag force. The fact that the difference is large at lowaltitude conditions suggests that the ion-electron drag force becomes more important as the density is increased.

As was shown in Sec. III, the plasma-optic regime does not provide effective plasma density reduction at low altitude. On the other hand, in the MHD regime, the $J \times B$ drift is an effective way to achieve plasma density reduction for low-altitude conditions.

The solution map in current density-length coordinate space is shown in Fig. 12. This solution map suggests possible current density and length ranges for plausible solution with strong plasma density reduction. While simulating the $E \times B$ layer, we set the current density to obtain conditions for strong plasma density reduction. In a practical situation, we cannot control the current density and instead the potential voltage drop is controlled. The larger length $L_{y}$ also gives high plasma density reduction. However, the effect of the plasma density reduction becomes saturated with length, as displayed in Fig. 12.

\section{Effect of Electrostatic Sheath Formation}

Another possibility to reduce electron density in the plasma layer is to form a high-voltage sheath near the negative electrode. In this case, electrons will be depleted from the sheath that will allow radio communication. This is based on the fact that electromagnetic waves interact primarily with plasma electrons. It was found recently that a magnetic field stabilizes a high-voltage sheath [26]. Thus, in this case, the magnetic field plays a secondary role in just creating proper conditions for high-voltage sheath formation, in contrast to previously described effects.

The magnetic field range considered in the preceding analyses is well below the magnitude required for ion magnetization. Therefore, in the steady state, the ions are accelerated toward the negative electrode by the electric field of the sheath without an effect of the magnetic field. The current density in the sheath can be calculated according to Child's law. When a negative voltage is applied to a substrate immersed in a plasma, electrons are repelled from the substrate, leading to sheath formation. Electrons drift away from the target across the magnetic field, due to the presence of the high electric field and rare collisions with ions and neutrals. In the steady state, the ions are then accelerated toward the substrate by the electric field of the sheath. In the one-dimensional steady-state case, the sheath thickness can be estimated as [26]

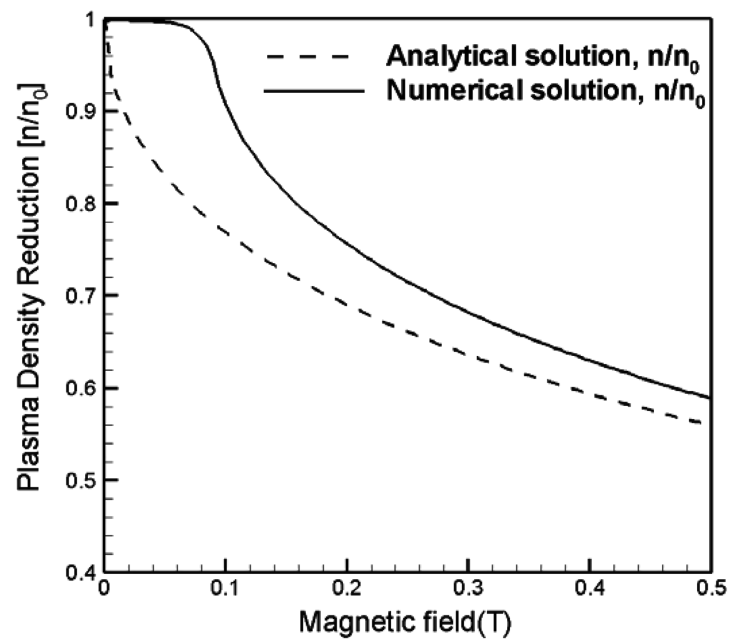

a)

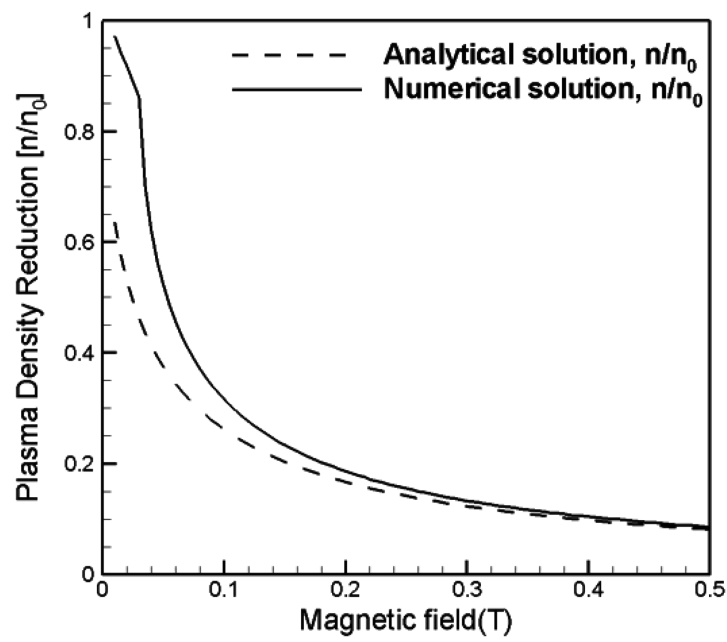

b)

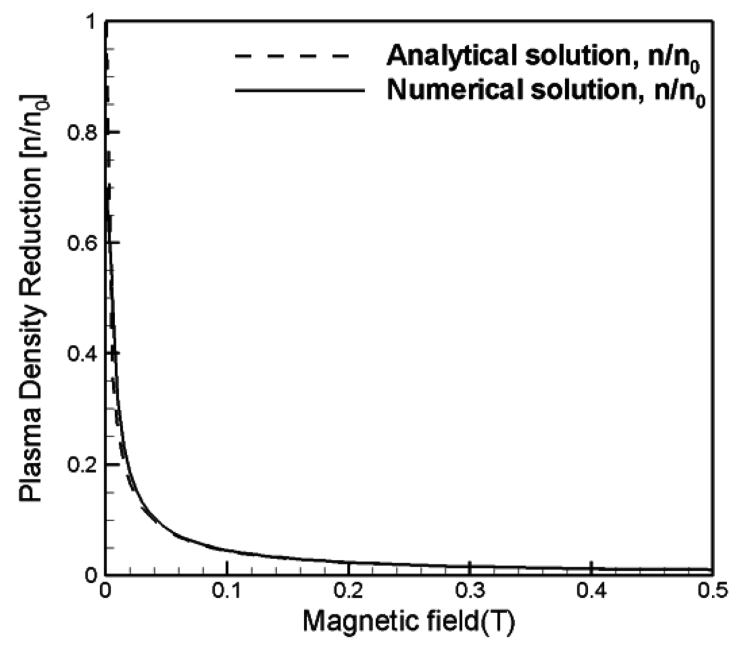

c)

Fig. 11 Analytical solution vs numerical solution for a) $41 \mathrm{~km}$ with current density of about $500 \mathrm{~A} / \mathrm{m}^{2}$ and distance between electrodes of about $10 \mathrm{~cm}$, b) $61 \mathrm{~km}$ with current density of about $500 \mathrm{~A} / \mathrm{m}^{2}$ and distance between electrodes of about $10 \mathrm{~cm}$, and c) $81 \mathrm{~km}$ with current density of about $500 \mathrm{~A} / \mathrm{m}^{2}$ and distance between electrodes of about $10 \mathrm{~cm}$. 


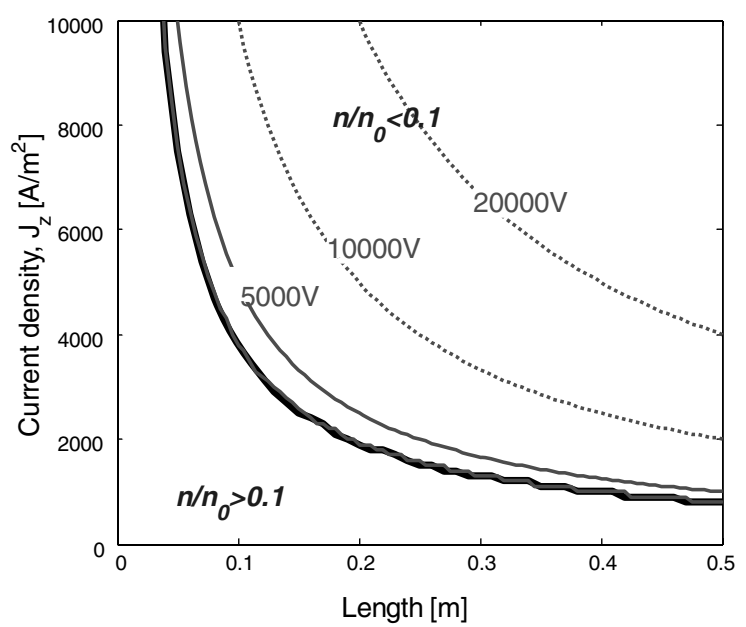

Fig. 12 Solution map for $41 \mathrm{~km}$. The solution is based on a MHD 1D analytical solution.

$$
s=\left(\frac{4}{9} \varepsilon\right)^{1 / 2}\left(\frac{2 Z_{i} e}{m_{i}}\right)^{1 / 4} \frac{U^{3 / 4}}{\left(e Z_{i} N V\right)^{1 / 2}}
$$

where $V$ is the ion velocity at the sheath edge, $U$ is the voltage across the sheath, $s$ is the sheath thickness, $e$ is the permittivity of vacuum, $Z_{i}$ is the ion mean charge number, $N$ is the plasma density at the sheath edge, and $m_{i}$ is the ion mass. It is obvious that the steady-state sheath thickness is determined by plasma density and ion velocity at the sheath edge for a given bias voltage.

The calculated sheath thickness is shown in Fig. 13 as a function of the applied voltage, with plasma density as a parameter. One can see that under certain conditions, the sheath thickness may be large enough to allow radio signal reception by the antenna.

It should be noted that the formation of the high-voltage sheath near the cathode can cause breakdown because the $E / N$ required is quite high. However, application of the magnetic field leads to highvoltage sheath stabilization. In fact, it was experimentally demonstrated that voltage up to $8 \mathrm{kV}$ can be maintained across the sheath [26].

In the future, we will consider a combination of the electromagnetic effect $(E \times B$ layer) and the electrostatic effect of sheath formation as an efficient plasma mitigation technique. In this case, one can expect that $E \times B$ plasma acceleration effect will contribute to plasma density reduction, which in turn will lead to larger sheath thickness. If sheath thickness becomes comparable with the size of the antenna, one can expect electromagnetic signal reception through the hypersonic plasma layer.

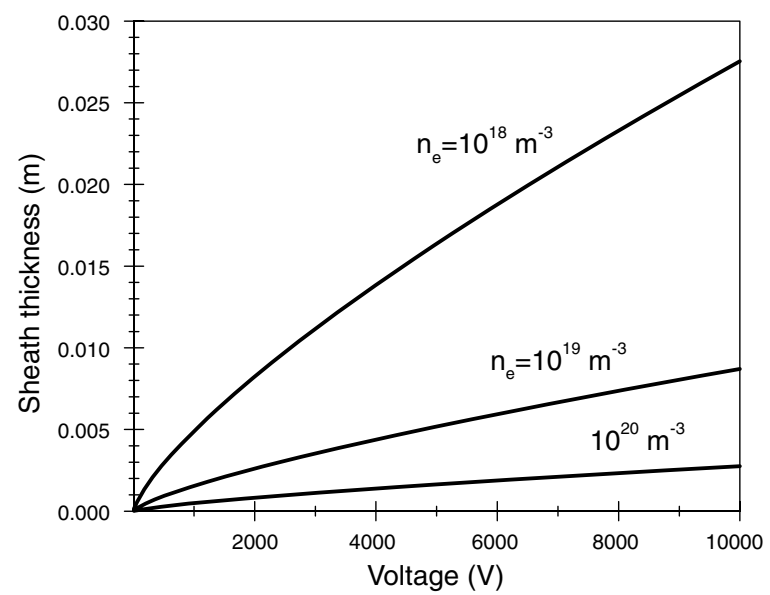

Fig. 13 Thickness of the electrostatic sheath with plasma density as a parameter.

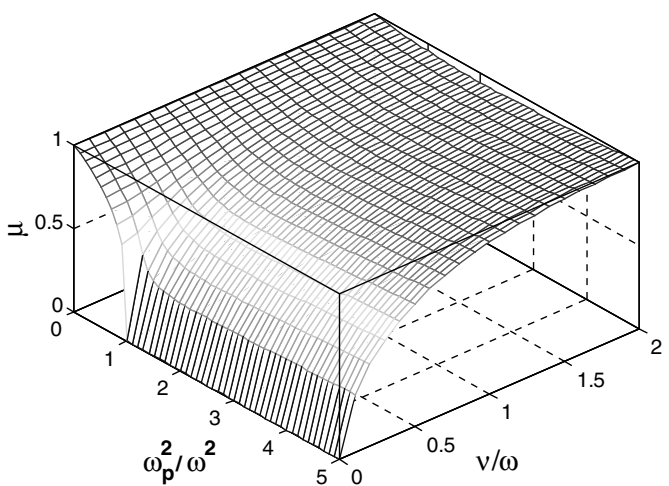

Fig. 14 Index of refraction along the plasma frequency and collision frequency.

\section{Wave Absorption Reduction}

In this section, we will estimate the benefits of the reduced plasma density in the plasma layer in terms of electromagnetic wave absorption through the magnetized plasma. For the plasma with collision frequency $v$, the radiowave attenuation can be expressed as [27]

$$
\kappa \propto \frac{1}{\mu} \frac{n \cdot v}{\omega^{2}+v^{2}} \mathrm{~dB} / \mathrm{m}
$$

We can assume that the collision frequency is proportional to the plasma density under typical conditions of the reentry flight. One can see from Eq. (29) that the radiowave attenuation depends on the index of refraction. The index of refraction leads to strong wave attenuation in the case of radio frequency close to the angular plasma frequency. This angular plasma frequency could be considered as a cutoff frequency for the reentry communication.

Figure 14 shows the index of refraction as a function of plasma frequency and collision frequency. The index of refraction decreases with increase of the plasma density under typical condition of reentry flight. Smaller refraction index leads to reduced radiowave attenuation, according to Eq. (29). For instance, in the case of $n=10^{15} \mathrm{~m}^{-3}$ and $160.5-\mathrm{Hz}$ radiowave, the wave attenuation is about $-22 \mathrm{~dB}$. When a magnetic field of about $1500 \mathrm{G}$ and potential of about $100 \mathrm{~V}$ are applied, the density reduction factor is about 0.05 and the radiowave attenuation is about $-7 \mathrm{~dB}$. Under the same magnetic field condition, if we increase the voltage up to $10 \mathrm{kV}$, the radiowave attenuation becomes below $-1 \mathrm{~dB}$.

\section{Conclusions}

It was proposed that application of electric and magnetic fields could lead to significant reduction of the plasma density, allowing radio communication through a hypersonic plasma layer. Specifically, an $E \times B$ crossed-field configuration is proposed and studied. Two regimes of $E \times B$ layer are considered: namely, the plasma-optic and MHD limits. Both analytical and numerical results suggest that significant reduction of the plasma density is possible at large altitudes. Electrostatic sheath formation is also analyzed and it was found that the electron-depleted region may have large thickness, comparable with the size of the antenna. Future work will include two-dimensional modeling to determine how much of the plasma area is affected by this system. In addition, an experimental work is underway to validate the model predictions.

\section{Acknowledgments}

The authors gratefully acknowledge the support of the U.S. Air Force in funding this work, through the Phase-II Small Business Innovation Research grant titled "ReComm- ReEntry and Hypersonic Vehicle Plasma Communications System," contract FA871806-C-0038. The authors wish to thank David Morris, Charles Jones, Jean-Luc Cambier, Chris Davis, Kristina Lemmer, and Jonathan Zagel for very useful discussions on this subject. 


\section{References}

[1] Bachynski, M. P., Propagation Factors in Space Communication, edited by W. T. Blackband, Technivision, Maidenhead, England, U.K., 1967.

[2] Usui, H., Matsumoto, H., Yamashita, F., Yamane, M., and Takenaka, S., "Computer Experiments on the Measurements of Reentry Plasma with Radio Waves," Active Experiments in Space Plasmas, Vol. 24, No. 8, 1999, pp. 1069-1072.

[3] Rybak, J. C., and Chirchill, R. J., "Progress in Reentry Communication," IEEE Transactions on Aerospace and Electronic Systems, Vol. aes-7, No. 5, 1971, pp. 879-894. doi:10.1109/TAES.1971.310328

[4] Leblanc, J. E., and Fujiwara, T., "Comprehensive Analysis of Communication with a Reentry Vehicle During Blackout Phase," Transactions of the Japan Society for Aeronautical and Space Sciences, Vol. 39, No. 124, 1996, pp. 211-221.

[5] Friel, P. J., and Rosenbaum, B., "Propagation of Electromagnetic Waves Through Reentry-Induced Plasmas," Advances in the Astronautical Sciences, edited by H. Jacobs, Vol. 11, American Astronautical Society, New York, 1963, p. 399.

[6] Huber, P. W., and Sims, T. E., "The Reentry Communication Problem," Astronautics and Aeronautics, Vol. 2, 1964, p. 30.

[7] Schexnauder, C. J., "Electron Density Reduction in Re-Entry Plasma Due to Nitrogen Atom Removal," AIAA Journal, Vol. 8, No. 2, 1970, pp. 375-377.

[8] Belov, I. F., Borovoy, V. Ya., Gorelov, V. A., Kireev, A. Y., Korolev, A. S., and Stepanov, E. A., "Investigation of Remote Antenna Assembly for Radio Communication with Reentry Vehicle," Journal of Spacecraft and Rockets, Vol. 38, No. 2, 2001, pp. 249-256.

[9] Nasarenko, S. V., Newell, A. C., and Zakharov, V. E., "Communication Through Plasma Sheath via Raman (Three Wave) Scattering Process," Physics of Plasmas, Vol. 1, No. 9, 1994, p. 2827 doi: $10.1063 / 1.870521$

[10] Hodara, H., "The Use of Magnetic Fields in the Elimination of the ReEntry Radio Blackout," Proceedings of the IRE, Vol. 49, Inst. of Radio Engineers, New York, 1961, p. 1825.

[11] Macheret, S. O., Shneider, M. N., and Miles, R. B., "Magnetohydrodynamic Control of Hypersonic Flows and Scramjet Inlets Using Electron Beam Ionization," AIAA Journal, Vol. 40, No. 1, 2002, pp. 7481.

[12] Keidar, M., and Beilis, I. I., "Electron Transport Phenomena in Plasma Devices with $E \times B$ Drift," IEEE Transactions on Plasma Science, Vol. 34, No. 3, 2006, pp. 804-814. doi:10.1109/TPS.2006.874852

[13] Akey, N. D., and Cross, A. E., "Radio Blackout Alleviation and Plasma Diagnostic Results from a 25,000 Foot per Second Blunt-Body Reentry," NASA TN D-5615, 1970.

[14] Grantham, W. L., "Flight Results of a 25,000 Foot per Second Reentry
Experiment Using Microwave Reflectometers to Measure Plasma Electron Density and Standoff Distance," NASA TN D-6062, 1970.

[15] Jones, W. L., and Cross, A. E., "Electrostatic Probe Measurements of Plasma Surrounding Three 25,000 Foot per Second Reentry Flight Experiments," NASA SP-252, 1970, p. 109.

[16] Huba, J. D., NRL Plasma Formulary, Naval Research Lab., Washington, D.C., 2000.

[17] Scalabrin, L. C., and Boyd, I. D., "Numerical Simulation of Weakly Ionized Hypersonic Flow for Reentry Configurations," AIAA Paper 2006-3773, June 2006.

[18] Boyd, I. D., "Modeling of Plasma Formation in Rarefied Hypersonic Entry Flows," AIAA Paper 2007-0206, January 2007.

[19] Keidar, M., Boyd, I. D., and Beilis, I. I., "Plasma Flow and Plasma-Wall Transition in Hall Thruster Channel," Physics of Plasmas, Vol. 8, No. 12 , 2001, pp. 5315-5322. doi:10.1063/1.1421370

[20] Keidar, M., Beilis, I. I., Boxman, R. L., and Goldsmith, S., "2D Expansion of the Low-Density Interelectrode Vacuum Arc Plasma Jet in an Axial Magnetic Field," Journal of Physics D: Applied Physics, Vol. 29, 1996, pp. 1973-1983. doi:10.1088/0022-3727/29/7/034

[21] Keidar, M., Boyd, I. D., and Beilis, I. I., "Modeling of a High-Power Thruster with Anode Layer," Physics of Plasmas, Vol. 11, No. 4, 2004, pp. 1715-1722. doi:10.1063/1.1668642

[22] Candler, G., and MacCormack, R. W., "Computation of Weakly Ionized Hypersonic Flows in Thermochemical Nonequilibrium," Journal of Thermophysics and Heat Transfer, Vol. 5, 1991, pp. 266273.

[23] Josyula, E., and Bailey, W. F., "Governing Equations for Weakly Ionized Plasma Flowfields of Aerospace Vehicles," Journal of Spacecraft and Rockets, Vol. 40, No. 6, 2003, pp. 845-857.

[24] Park, C., "Nonequilibrium Hypersonic Aerodynamics," Wiley, New York, 1990.

[25] Candler, G. V., and MacCormack, R. W., "The Computation of Hypersonic Ionized Flows in Chemical and Thermal Nonequilibrium," AIAA Paper 88-0511, 1988.

[26] Keidar, M., Monteiro, O. R., Anders, A., and Boyd, I. D., "Magnetic Field Effect on the Sheath Thickness in Plasma Immersion Ion Implantation," Applied Physics Letters, Vol. 81, No. 7, 2002, pp. 1183 1185 . doi:10.1063/1.1499516

[27] Christopher, Paul, "Millimeter-Wave Communications for Atmospheric Re-Entry Vehicles," Proceedings of SPIE, Vol. 3232, 1998, pp. 21-32.

A. Ketsdever Associate Editor 